\title{
On performance limitations and property correlations of Al-doped ZnO deposited by radio-frequency sputtering
}

Paper

Crovetto, Andrea; Ottsen, Tobias Sand; Stamate, Eugen; Kjær, Daniel; Schou, Jørgen; Hansen, Ole

Published in:

Journal of Physics D: Applied Physics

Link to article, DOI:

$10.1088 / 0022-3727 / 49 / 29 / 295101$

Publication date:

2016

Document Version

Peer reviewed version

Link back to DTU Orbit

Citation (APA):

Crovetto, A., Ottsen, T. S., Stamate, E., Kjær, D., Schou, J., \& Hansen, O. (2016). On performance limitations and property correlations of Al-doped $\mathrm{ZnO}$ deposited by radio-frequency sputtering: Paper. Journal of Physics $D$ : Applied Physics, 49(29), [295101]. https://doi.org/10.1088/0022-3727/49/29/295101

\section{General rights}

Copyright and moral rights for the publications made accessible in the public portal are retained by the authors and/or other copyright owners and it is a condition of accessing publications that users recognise and abide by the legal requirements associated with these rights.

- Users may download and print one copy of any publication from the public portal for the purpose of private study or research.

- You may not further distribute the material or use it for any profit-making activity or commercial gain

- You may freely distribute the URL identifying the publication in the public portal 


\title{
On performance limitations and property correlations of Al-doped $\mathrm{ZnO}$ deposited by radio-frequency sputtering
}

\author{
Andrea Crovetto ${ }^{1, *}$, Tobias Sand Ottsen ${ }^{1}$, Eugen Stamate ${ }^{2}$, \\ Daniel Kjær ${ }^{1,3}$, Jørgen Schou ${ }^{4}$, and Ole Hansen ${ }^{1,5}$ \\ ${ }^{1}$ DTU Nanotech, Technical University of Denmark, DK-2800 Kgs. Lyngby, Denmark \\ 2 DTU Energy, Technical University of Denmark, DK-4000 Roskilde, Denmark \\ ${ }^{3}$ CAPRES A/S, Scion-DTU, DK-2800 Kgs. Lyngby, Denmark \\ ${ }^{4}$ DTU Fotonik, Technical University of Denmark, DK-4000 Roskilde, Denmark \\ ${ }^{5}$ CINF, Center for Individual Nanoparticle Functionality, Technical University of \\ Denmark, DK-2800 Kgs. Lyngby, Denmark \\ E-mail: ancro@nanotech.dtu.dk
}

\begin{abstract}
The electrical properties of $\mathrm{RF}$-sputtered $\mathrm{Al}$-doped $\mathrm{ZnO}$ are often spatially inhomogeneous and strongly dependent on deposition parameters. In this work, we study the mechanisms that limit the minimum resistivity achievable under different deposition regimes. In a low- and intermediate pressure regime, we find a generalized dependence of the electrical properties, grain size, texture, and $\mathrm{Al}$ content on compressive stress, regardless of sputtering pressure or position on the substrate. In a high-pressure regime, a porous microstructure limits the achievable resistivity and causes it to increase over time as well. The primary cause of inhomogeneity in the electrical properties is identified as energetic particle bombardment. Inhomogeneity in oxygen content is also observed, but its effect on the electrical properties is small and limited to the carrier mobility.
\end{abstract}




\section{Introduction}

Transparent conductive oxides (TCO) are thin-film materials that feature low electrical resistivity and high optical transmission in the visible region of light, which are requirements in applications such as flat panel displays, low emissivity coatings, and top electrodes in thin-film solar cells [1]. Currently, the best-performing TCO is Sndoped $\mathrm{In}_{2} \mathrm{O}_{3}$ (ITO), which, however, suffers from the high cost and limited availability of indium [2]. This can be a problem for large-area applications such as photovoltaics. Therefore, Al-doped $\mathrm{ZnO}$ (AZO) has been widely used in research and industry as a top electrode in thin-film solar cells based on amorphous silicon or $\mathrm{Cu}(\mathrm{In}, \mathrm{Ga}) \mathrm{Se}_{2}$, which have recently reached more than $22 \%$ power conversion efficiency on the laboratory scale [3]. The main advantage of AZO over ITO is the greater abundance and lower cost of its component elements. AZO thin film deposition by RF- or DC magnetron sputtering technique combines high film quality with relatively high deposition rate, compatibility with large-area substrates, and is routinely employed in thin-film solar cell production $[4,5]$. Resistivity in the $10^{-4} \Omega \mathrm{cm}$ range can be obtained by sputtering while retaining more than $80 \%$ average transmission in the visible region of light [6]. However, an issue related to sputter deposition of AZO is that the resistivity of the deposited films exhibits spatial inhomogeneity $[7,8,9,10,11,12,13]$. Mainly two explanations exist for this phenomenon: 1) bombardment of the film by inhomogeneously distributed energetic particles during deposition [7]; 2) inhomogeneity in the amount and activity of oxygen reaching the substrate, which results in non-optimal oxygen stoichiometry in certain regions of the film [8].

According to hypothesis (1), $\mathrm{O}^{-}$and $\mathrm{O}_{2}^{-}$ions (the former being more abundant) $[9,14,15]$ are formed at the target and accelerated through the cathode sheath up to an energy corresponding to the target DC bias voltage. Upon leaving the cathode sheath, such a collimated beam of energetic ions travels mostly perpendicular to the target surface with a small collision cross section with the working gas [16]. In the plasma, $\mathrm{O}^{-}$ions may undergo electron detachment and turn into equally energetic $\mathrm{O}$ neutrals [17]. Film bombardment by such energetic species leads to formation of crystal defects and deteriorates the electrical properties.

According to hypothesis (2), excess oxygen reaches the substrate in the regions of largest plasma density and causes the resistivity to increase or decrease in those regions, depending on whether the excess oxygen results in an oxygen content in the film that is above or below optimal, respectively [8, 18].

In an attempt to distinguish which mechanisms limit the minimum achievable resistivity under different deposition conditions, we systematically examine the spatial distribution of the electrical, structural, morphological, and compositional properties of RFsputtered AZO at different deposition pressures. This investigation method also allows to discriminate between property correlations that only exist under specific conditions, and correlations of a more general nature. 


\section{Experimental details}

Thin AZO films were deposited by RF magnetron sputtering with a balanced cathode and a hot-pressed ceramic target (Kurt J. Lesker $\mathrm{ZnO} / \mathrm{Al}_{2} \mathrm{O}_{3}$ 98/2 wt\%, 99.99\% purity). The target had a circular shape with a diameter of $50.8 \mathrm{~mm}$, a thickness of $3.2 \mathrm{~mm}$, and was indium-bonded to a $\mathrm{Cu}$ backing plate. The experiments in this work were carried out when the depth of the erosion track on the target was approximately $0.2 \mathrm{~mm}$. At the target surface, the component of the magnetic flux density along the $x$ direction (Fig. 1) was approximately $57.5 \mathrm{mT}$.

One Si and one quartz substrate (size: $10 \mathrm{~mm} \times 30 \mathrm{~mm}$ each) were directly facing the target at a target-substrate distance of $30 \mathrm{~mm}$ and kept at ground potential (Fig. 1). The two types of substrate were chosen for compatibility with the characterization techniques used in this work.

The chamber was pumped down to a base pressure of $5 \times 10^{-7}$ Torr by a turbo pump, then pure Ar gas (99.999\%) was introduced and the discharge RF power was set to $20 \mathrm{~W}$ with a $0.25 \mathrm{~W} / \mathrm{s}$ ramp. The target was pre-sputtered at the desired deposition pressure by closing a shutter between the target and the substrate. In the meantime, the substrate stage was heated to $150^{\circ} \mathrm{C}$. After pre-sputtering for 15 minutes, the shutter was opened and film deposition took place. The measured target self-bias varied between $132 \mathrm{~V}$ (at $2.5 \mathrm{mTorr}$ ) and $146 \mathrm{~V}$ (at $15 \mathrm{mTorr}$ ). The temperature remained between 145 and $155^{\circ} \mathrm{C}$ during deposition, as measured by a thermocouple. After deposition, the substrates were allowed to cool down naturally in vacuum, and they were taken out when the measured temperature was below $50^{\circ} \mathrm{C}$. The only parameter that was changed from one deposition to another was the deposition pressure. In the following sections, pressures of the order of 2.5 mTorr, 5 mTorr, and 15 mTorr are referred to as low-, intermediate-, and high pressure regimes respectively.

We show different properties of the deposited films as a function of distance from the central symmetry axis of the magnetron $x=0$ (labeled $x_{0}$ ) through the point directly facing the erosion track of the target at a radial distance $x=1.2 \mathrm{~cm}$ (labeled $x_{e}$ ) and up to the end of the substrate, at $x=3 \mathrm{~cm}$ (labeled $x_{3}$ ). Because the sputtering setup is center-symmetric, we also expect the film properties to be center-symmetric. This was confirmed by measuring thickness and resistivity on a film deposited on a $6 \mathrm{~cm}$-long quartz substrate centered on $x=0$. Hence, the distribution of properties is in fact a "radial" distribution instead of simply a "spatial" distribution.

The AZO/Si films were imaged with a scanning electron microscope (SEM) equipped with a field emission gun and an in-lens detector (FE-SEM, Supra 60VP, Zeiss). Energy dispersive X-ray spectroscopy (EDX) was performed in the same instrument on the $\mathrm{AZO} / \mathrm{Si}$ films using a $\mathrm{Si}$ drift detector $\left(\mathrm{X}-\mathrm{Max}^{N} 50\right.$, Oxford Instruments) and a beam voltage of $3 \mathrm{kV}$. If the beam voltage was higher, artifacts in the composition appeared due to x-ray generation in the $\mathrm{Si}$ substrate. On the other hand, strong $\mathrm{Zn}, \mathrm{O}$, and $\mathrm{Al}$ $\mathrm{x}$-ray peaks are present at energies well below $3 \mathrm{keV}$.

$\mathrm{X}$-ray diffraction (XRD) patterns were collected on the AZO/quartz films with a 


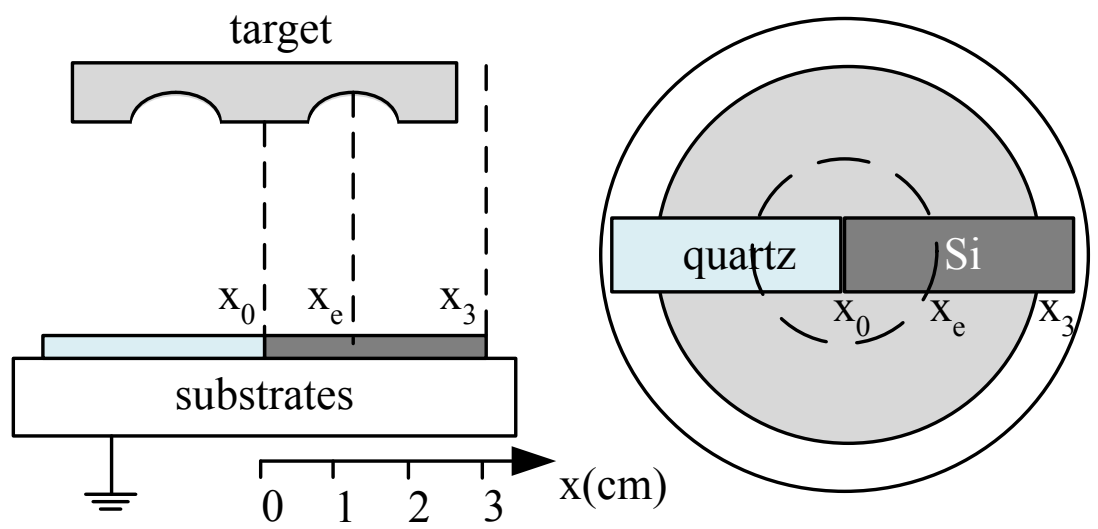

Figure 1. Schematic drawing of the sputtering geometry and the position of the substrates with respect to the target. All dimensions are to scale. $x_{0}$ is the symmetry axis of the magnetron, $x_{e}$ is the radial distance corresponding to the maximum depth of the erosion track of the target. $x_{3}$ is the edge of the substrate.

Bruker D8 Discover diffractometer equipped with a Göbel mirror for a parallel primary beam. The measurement was performed in Bragg-Brentano configuration using $\mathrm{Cu}-\mathrm{K}_{\alpha}$ radiation, a $0.001^{\circ}(2 \theta) / \mathrm{s}$ acquisition time, a $0.6 \mathrm{~mm}$ primary slit, a $0.6 \mathrm{~mm}$ anti-scatter slit, and a $0.2 \mathrm{~mm}$ detector slit. The slit sizes were selected in order to obtain as low instrumental broadening as possible, while still being able to detect a clear (002) peak. Instrumental broadening was measured using a standard $\mathrm{LaB}_{6}$ sample. Out-of plane crystallite size and micro-strain were calculated by fitting the (002) peak with a Pseudo-Voigt function (i.e. a weighted sum of a Lorentzian and a Gaussian function) and by extracting the parameters of the Lorentzian and Gaussian contributions after subtracting instrumental broadening. The detailed procedure is described elsewhere [19]. The out-of plane lattice shift $\varepsilon$ was measured based on the position of the (002) XRD peak. In-plane stress $\sigma$ was calculated from the measured lattice shift using a biaxial stress model and literature values for the $\mathrm{ZnO}$ stress tensor elements [13]. The thickness and optical functions of the AZO/Si films were measured in reflection mode in the spectral range $0.7-5.9 \mathrm{eV}$ on a rotating compensator spectroscopic ellipsometer (M-2000, J.A. Woollam Co.) using a collimated beam with a spot size of approximately $200 \mu \mathrm{m} \times 300 \mu \mathrm{m}$. Ellipsometry spectra were analyzed and fitted with the CompleteEase software package (version 5.06 - J.A. Woollam Co.).

Sheet resistance was measured on the AZO/quartz films with a four-point probe with electrode spacing of $0.5 \mathrm{~mm}$. The carrier concentration and Hall mobility were measured on the AZO/quartz films with an industrial micro-Hall setup (CAPRES A/S) including an equidistant, collinear micro 7-point probe with electrode spacing of $10 \mu \mathrm{m}$. The measurement principle and data analysis procedure have been described elsewhere $[20,21]$. 


\section{Results}

In this section we present the radially-resolved measurement of a few relevant properties of AZO films deposited at different pressures. The average of the radial measurements is also plotted against deposition pressure, in the cases where it provides useful information. The electrical properties are presented first, followed by other properties that provide insights into the mechanisms limiting the minimum achievable resistivity, or that may be directly correlated to the electrical properties.

\subsection{Resistivity}

Fig. 2(a) shows that the resistivity of AZO is strongly dependent on deposition pressure, with the films exhibiting significant loss of electrical quality both in the low- and in the high pressure regime. The radial standard deviation of resistivity for each of our deposited films follows a similar trend as the average resistivity, i.e. with a minimum at intermediate pressure. Therefore, the films deposited at the optimal pressure achieve both the lowest resistivity and the lowest inhomogeneity. The radial distribution of electrical resistivity for films deposited at different pressures is shown in Fig. 2(b). In all films, the resistivity has a maximum at $x_{0}$ and a minimum at $x_{3}$. Furthermore, we note that only the film deposited in the high-pressure regime experiences significant degradation in resistivity (approximately by a factor of 5) over a storage period of 7 months. This has been observed in previous studies on AZO sputtered at high pressure by single-point measurements [22, 23]. From Fig. 2(b), we can conclude that resistivity degradation over time in samples deposited at a high pressure is a global phenomenon, i.e. it occurs to a similar extent at different positions of the film, and must be therefore related to a global property typical of the high-pressure films.

\subsection{Carrier concentration and mobility}

By inspection of Fig. 3, it is clear that the radial resistivity gradient is due to a gradient in both carrier concentration and mobility, which have a minimum at $x_{0}$ at all pressures. However, such gradients depend strongly on deposition pressure. For example, the mobility gradient in the low-pressure regime is much greater than in the other regimes. Note that mobility in the 2.5 mTorr sample near $x_{0}$ is even lower than on the other points on the same film, but such low mobilities cannot be measured with our setup due to their weak Hall signal. In contrast, in the high-pressure regime there is a weaker mobility gradient but the carrier concentration reaches by far the lowest values obtained in our experiments. Interestingly, a local mobility maximum is consistently found in the intermediate pressure regime at positions close to $x_{e}$. Its origin will be discussed in the following sections. 

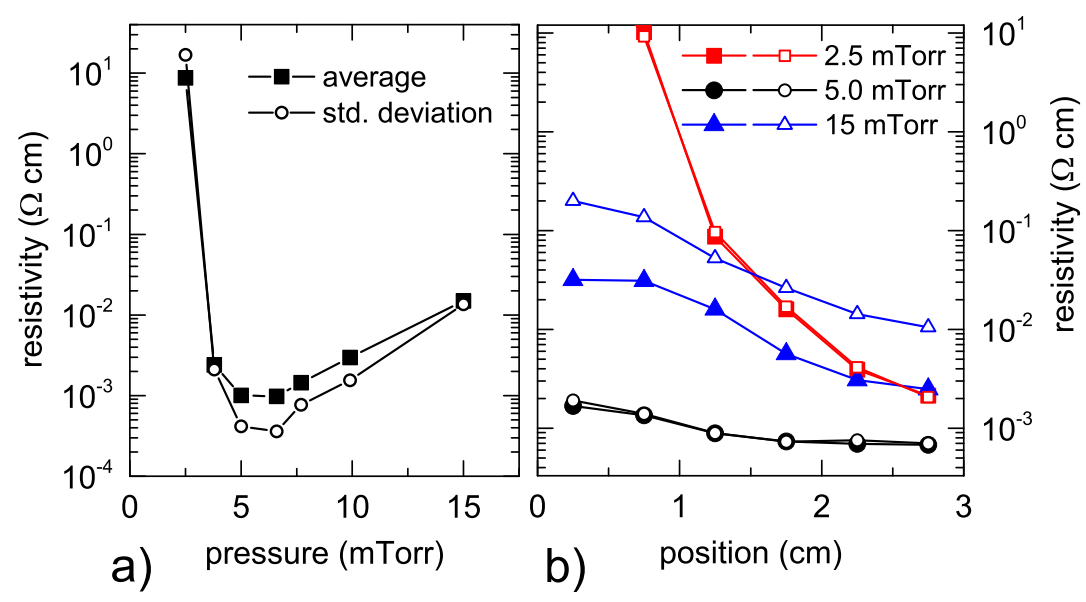

Figure 2. (a) Radially-averaged resistivity between $x_{0}$ and $x_{3}$, and its inhomogeneity (expressed as the standard deviation of resistivity between $x_{0}$ and $x_{3}$ ) versus deposition pressure. (b) Radial distribution of resistivity from $x_{0}$ to $x_{3}$. A first measurement was taken within one day after depositing each film (closed symbols); a second measurement was taken after storage for 7 months at atmospheric pressure and room temperature (open symbols).
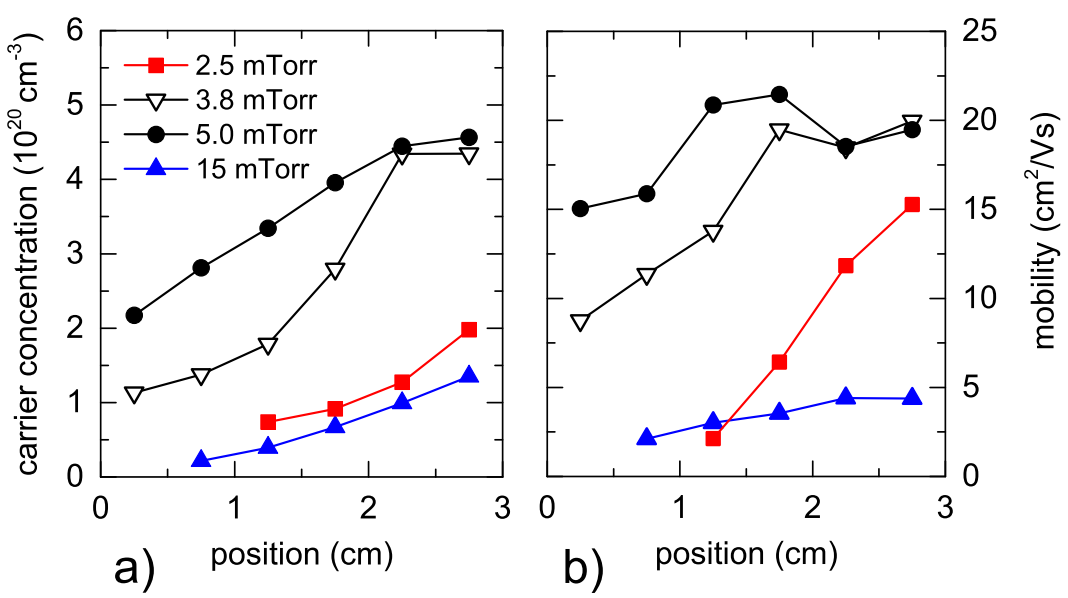

Figure 3. Radial distribution of carrier concentration and mobility from $x_{0}$ to $x_{3}$. Some data points are missing because mobilities lower than $\sim 2 \mathrm{~cm}^{2} / \mathrm{Vs}$ could not be measured with our Hall setup.

\subsection{Deposition rate}

A maximum in the spatially-averaged deposition rate (Fig. 4(a)) is found at about 5 mTorr, corresponding to the intermediate pressure regime. This resembles the trend found in a previous study on certain sputter-deposited oxides [24], where two mechanisms limiting the deposition rate were identified. In the high pressure regime, the deposition rate decreased with pressure due to thermalization of the sputtered species, as predicted by a general model of sputter deposition [25]. In the low pressure regime the deposition rate dropped due to resputtering of the growing film caused by energetic ion bombardment. The (re)sputtering threshold of $\mathrm{ZnO}$ has been estimated to be 33- 


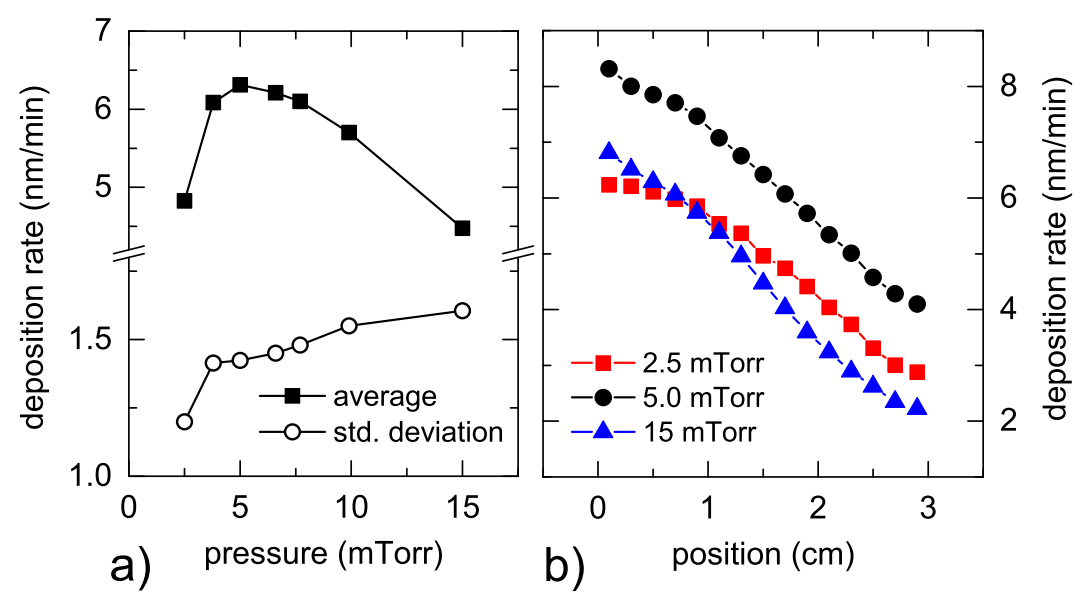

Figure 4. (a) Radially-averaged deposition rate between $x_{0}$ and $x_{3}$, and its inhomogeneity (expressed as the standard deviation of deposition rate between $x_{0}$ and $x_{3}$ ) versus deposition pressure. (b) Radial distribution of deposition rate from $x_{0}$ to $x_{3}$.

$35 \mathrm{eV}$ by semi-empirical formulas [10] and numerical simulation [26]. Typical energy distributions of the $\mathrm{O}^{-}$ions impinging on growing AZO films in an $\mathrm{RF}$ discharge have been measured before [27] and are well above such a threshold. Due to a global drop in deposition rate in the low-pressure regime (Fig. 4), we conclude that our AZO film is partially resputtered by energetic particle bombardment in the low-pressure regime. Previous studies have shown that not only can resputtering effects exist in AZO, but they can also be spatially distributed: the regions of the film where a higher flux density of energetic ions is expected may have a higher resputtering rate [28, 29]. By inspection of the radial distribution of deposition rate in our films (Fig. 4(b)), we notice that the deposition rate profile flattens considerably near $x_{0}$ in the low-pressure regime, and a cross-over with the high-pressure regime curve occurs. This may be attributed to a higher resputtering rate near $x_{0}$.

\subsection{Lattice shift and stress}

Besides a drop in deposition rate from resputtering effects, energetic particle bombardment is expected to give rise to compressive stress due to the atomic peening mechanism [30]. According to quantitative models verified against a number of sputterdeposited materials [31], the in-plane compressive stress $\sigma$ scales with the product of the flux density of the bombarding particles $\Gamma$ times the square root of their kinetic energy $E$, that is, $\sigma \propto \Gamma \sqrt{E}$. This relation must be rewritten for RF-sputtered AZO, because the $\mathrm{O}^{-}$flux is not monoenergetic, but it has an energy distribution function centered on the energy corresponding to the target self-bias voltage (about $140 \mathrm{~V}$ in our study) and is rather uniformly distributed from $0 \mathrm{eV}$ up to approximately twice the value of the self-bias $[9,27]$. Also, both $\Gamma$ and $E$ may depend on deposition pressure $p$ and radial 

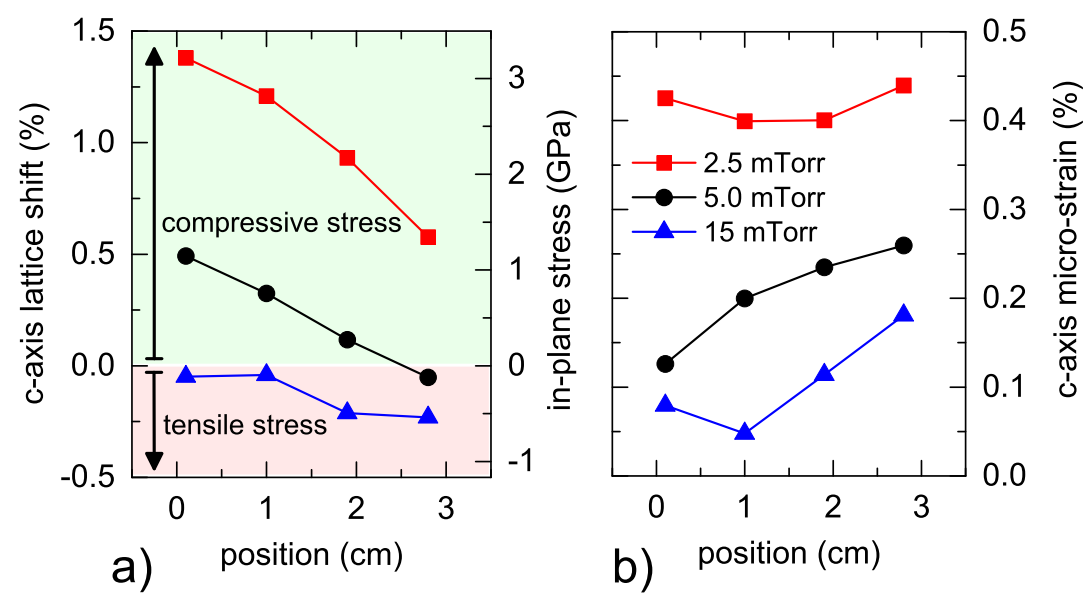

Figure 5. (a) Radial distribution of out-of-plane lattice shift from $x_{0}$ to $x_{3}$. The shift is calculated based on the position of the fitted (002) XRD peak at each radial position and expressed as percentage of the lattice constant of a reference $\mathrm{ZnO}$ powder (JCPDS card 36-1451). A positive lattice shift stands for lattice expansion in the outof-plane direction (compressive in-plane stress); a negative lattice shift signifies lattice compression (tensile in-plane stress). In-plane stress has been calculated based on a biaxial stress model with reference $\mathrm{ZnO}$ tensor elements [13].

(b) Radial distribution of root-mean-square micro-strain from $x_{0}$ to $x_{3}$ as determined by XRD line profile analysis after deconvoluting instrument- and size-related broadening effects. The micro-strain is expressed as percentage of the lattice constant of a reference $\mathrm{ZnO}$ powder (JCPDS card 36-1451).

position $x$. Hence, we generalize the empirical formula [31] as

$$
\sigma(p, x) \propto \int_{E_{\min }}^{E_{\max }} \Gamma(E, p, x) d\left(E^{1 / 2}(p, x)\right) \equiv \Sigma_{E^{1 / 2}}(p, x)
$$

where $\Sigma_{E^{1 / 2}}$ is introduced by definition and is the flux density integrated with respect to the square root of the kinetic energy at pressure $p$ and position $x$. In the following, we refer to it as "energy flux" for simplicity, and will assume our measured lattice shift to be roughly proportional to it. This proportionality can be justified experimentally for sputter-deposited AZO based on the results of a recent study, where the measured energy-weighted $\mathrm{O}^{-}$ion flux density and the measured lattice shift were found to be correlated for both DC- and RF sputtering [9].

The measured lattice shift and calculated stress of our AZO films in the three pressure regimes along the radial direction are shown in Fig. 5(a). In a biaxial stress model, a positive lattice shift $\varepsilon$ in the out-of-plane direction (lattice expansion) corresponds to compressive in-plane stress, whereas a negative out-of-plane lattice shift (lattice compression) corresponds to tensile in-plane stress. In our films there are two tendencies: 1) the lattice shift increases by decreasing deposition pressure, and 2) the lattice shift increases by decreasing radial position from $x_{3}$ to $x_{0}$. As a consequence, inplane stress in the films varies between the two extreme cases of a moderate tensile stress (-0.5 GPa at $15 \mathrm{~m}$ Torr at $\left.x_{3}\right)$ and a large compressive stress $(3.2 \mathrm{GPa}$ at $2.5 \mathrm{mTorr}$ at $\left.x_{0}\right)$. The transition from tensile to compressive stress from high- to low pressure regime 
is not unique to AZO, but is well known and quite general for sputter-deposited films [32]. These trends suggest that the energy flux, and the consequential lattice shift, are diminished both by increasing the deposition pressure and by moving away from $x_{0}$. We will demonstrate quantitatively in the next sections that this is uniquely reflected in the electrical properties of the films.

\subsection{Micro-strain}

Only very few works $[33,34,35]$ have shown the root-mean square (RMS) micro-strain in AZO films. This quantity is fundamentally different from the (macroscopic) strain discussed in the previous section. The micro-strain is the Gaussian-distributed variation of the lattice constant centered on the macroscopically-strained lattice constant of the material. From the radially-resolved measurement of micro-strain in Fig. 5(b) it is noted that the micro-strain globally increases as the pressure decreases. This is compatible with the higher probability of lattice dislocations and substitutional defects occurring in the low-pressure regime due to particle bombardment [30,31] and it is also consistent with the increased $\mathrm{Al}$ content in the low-pressure regime (Fig. 6(b)). When doping $\mathrm{ZnO}$ with $2 \% \mathrm{Al}$, the latter is expected to substitute $\mathrm{Zn}$ in a small number of $\mathrm{Zn}$ sites (the absolute maximum is $4 \%$ of the $\mathrm{Zn}$ sites assuming $100 \%$ dopant activation). Because $\mathrm{Al}^{3+}$ has a smaller ionic radius than $\mathrm{Zn}^{2+}$ (53 pm and $72 \mathrm{pm}$ respectively), the consequence of $\mathrm{Al}$ substitution should be that the $\mathrm{ZnO}$ lattice becomes strained in the vicinity of the Al lattice site. This should result in micro-strain instead of an overall lattice shift. The fact that different physical mechanisms may lie behind these two distinct types of strain is confirmed by the fact that in Fig. 5 there is no obvious relationship between the overall lattice shift and the micro-strain.

\subsection{Elemental composition}

In order to obtain excellent electrical properties in AZO films, it is crucial that the oxygen stoichiometry be optimal [36]. Amount and activation of the dopant $\mathrm{Al}$ is equally important $[37,38]$. Before analyzing our compositional results, however, two warnings must be expressed: 1) a systematic error around $\pm 5 \%$ (relative) should be expected for the absolute composition ratios, due to the relatively low beam voltage and use of elemental standards in EDX analysis; 2) a random error around $\pm 0.1 \%$ point (absolute) should also be expected, due to reproducibility limits of the EDX measurement. Since the deviations in atomic composition found in this study are always contained within a $1 \%$ point (absolute) range or less, care must be taken in interpreting weak trends in the radially-resolved measurements. From Fig. 6(a), we note that the global Al content increases and the $\mathrm{Zn}$ content decreases in the low pressure regime. In fact, resputtering effects in AZO films have been shown in previous work to be associated to preferential removal of Zn from the growing film [29]. As previously observed from Fig. 4, the largest resputtering rate in our films occurs in the low-pressure regime, which is consistent with the $\mathrm{Zn}$ depletion measured in the that regime. Al enrichment will be discussed in the 

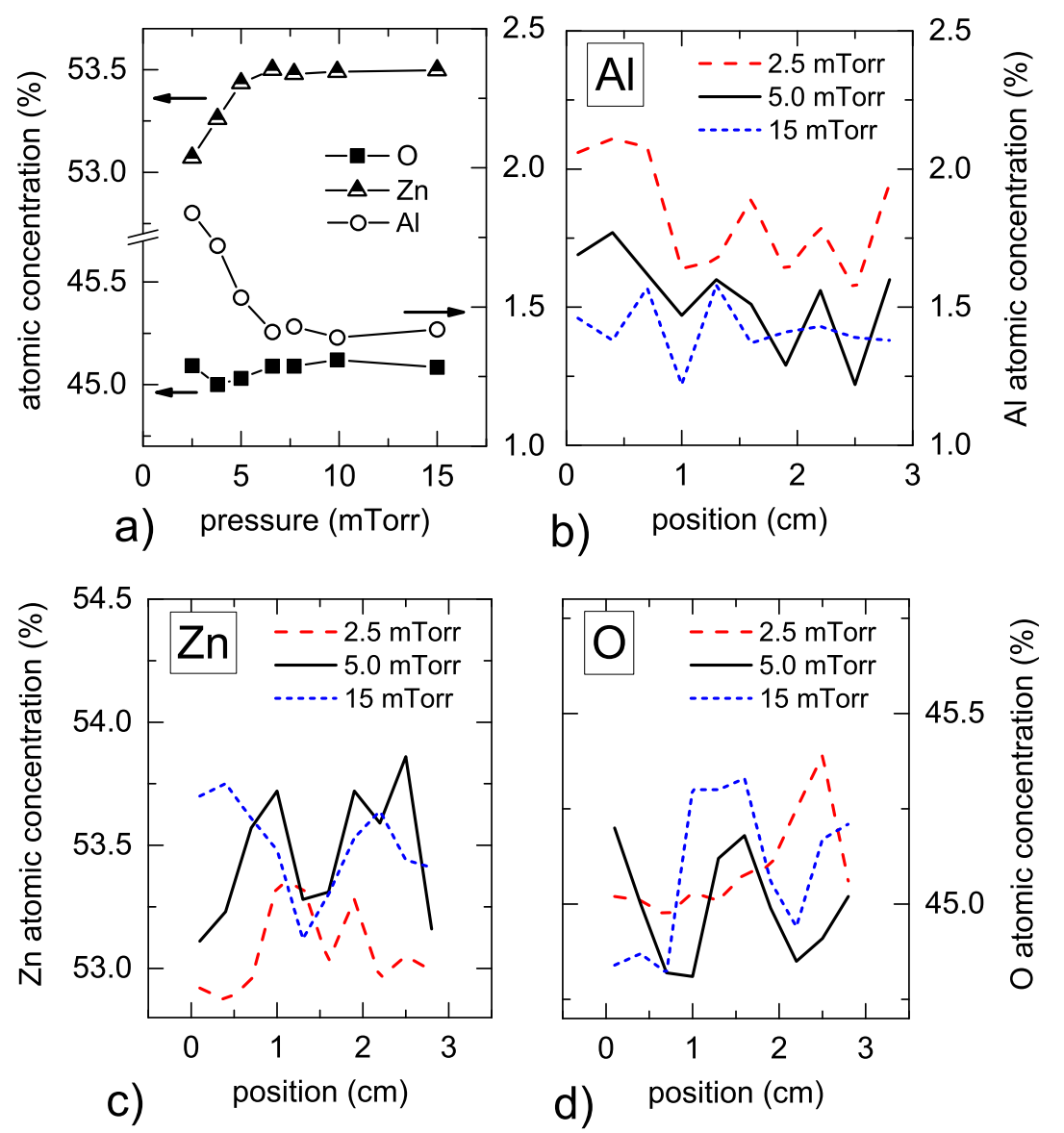

Figure 6. (a) Radially-averaged atomic composition versus deposition pressure measured by EDX. (b,c,d) Radially-resolved atomic composition of $\mathrm{Al}, \mathrm{Zn}$, and $\mathrm{O}$ from $x_{0}$ to $x_{3}$ measured by EDX.

following sections.

Even though the $\mathrm{O}$ content is roughly constant against pressure on an average level, $\mathrm{O}$ concentration exhibits a local maximum in the radially-resolved measurement at all pressures (Fig. 6(d)). The maximum moves progressively from $x_{e}$ to $x_{3}$ as the deposition pressure is decreased. We suggest that local maxima of the $\mathrm{O}$ content near $x_{e}$ may be related to the local maxima in Hall mobility near $x_{e}$ observed in Fig. 3(b). In fact, the electron mobility of AZO is known to exhibit a maximum in correspondence to the optimal oxygen content [36]. Besides that, no other measured quantity has a local maximum or minimum near $x_{e}$ in our study. Finally, we note that it is difficult to establish any trends in the $\mathrm{Zn}$ and $\mathrm{Al}$ content against radial position (the dips in $\mathrm{Zn}$ content near $x_{e}$ can be attributed to the local increase in $\mathrm{O}$ content).

\subsection{Morphology}

To investigate the influence of deposition pressure and radial position on film morphology, we examine SEM images in top- and cross sectional view of three 
$2.5 \mathrm{mTorr}$

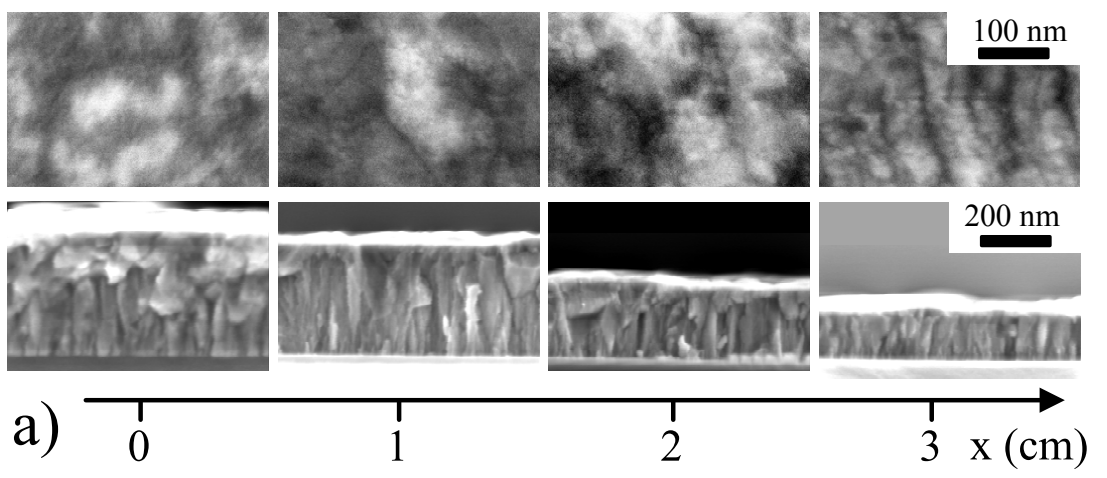

5.0 mTorr
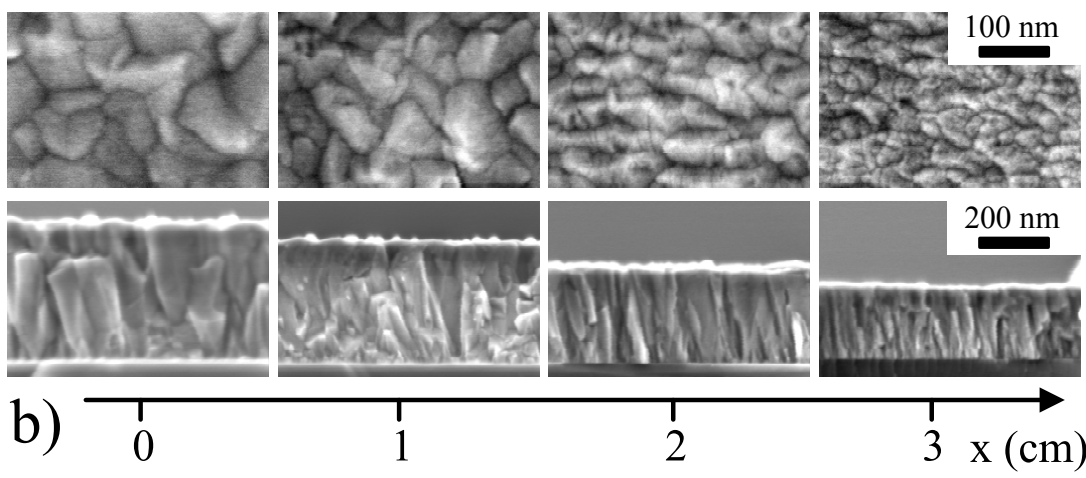

15 mTorr

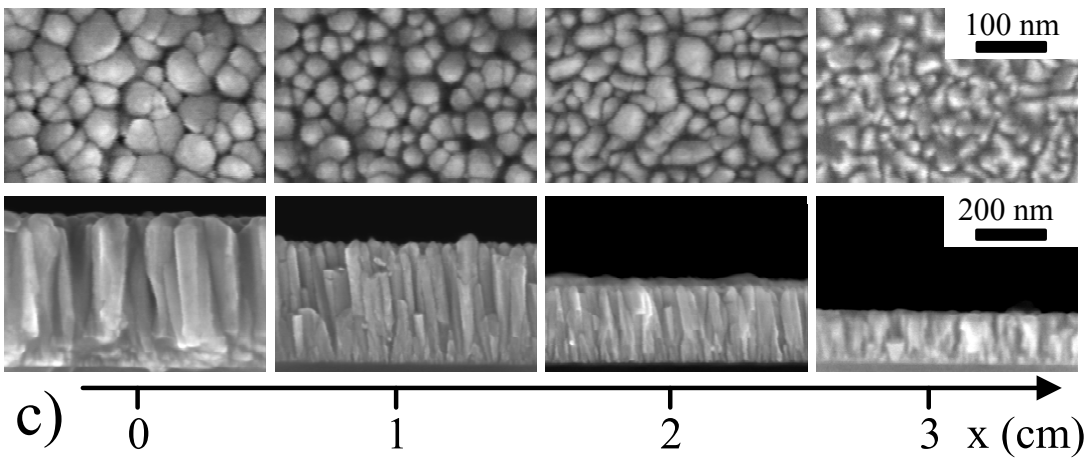

Figure 7. SEM images in top- and cross-sectional view as a function of radial position from $x_{0}$ to $x_{3}$ at different deposition pressures. (a) 2.5 mTorr, (b) 5.0 mTorr (c) 15 mTorr. The magnification is kept constant from left to right on each row.

representative films (Fig. 7). Interestingly, the cross-sectional images of the 2.5 mTorr film demonstrate that a phase with different morphology exists at the film top surface. This effect is greatly enhanced in the region near $x_{0}$ where, in the whole upper half of the film, columnar grains are replaced by a more homogeneous layer of nearly amorphous appearance. In fact, the XRD pattern of the 2.5 mTorr film near $x_{0}$ (not shown) has a very weak broad background hump around the (002) peak position, which is probably related to this nearly amorphous AZO phase. In the top-view images of the $2.5 \mathrm{mTorr}$ film, the crystal grains are not as sharply defined as in the other films, probably because those grains do not extend all the way up to the film surface. As noted in previous 

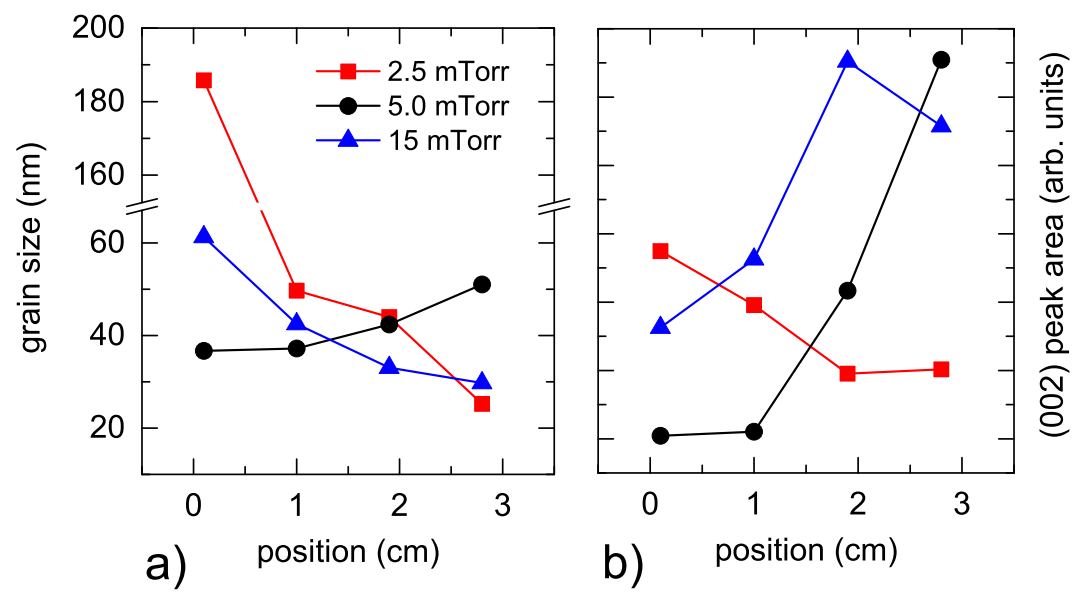

Figure 8. (a) Radial distribution of out-of-plane grain size from $x_{0}$ to $x_{3}$ as determined by XRD line profile analysis after deconvoluting instrument- and strainrelated broadening effects.

(b) Radial distribution of (002) peak area divided by film thickness at each point from $x_{0}$ to $x_{3}$. As a rough approximation, the (002) peak area is proportional to the degree of preferential c-axis orientation perpendicular to the substrate plane.

reviews, heavy particle bombardment is known to be responsible for film amorphization effects [39, 40].

On the other hand, in the high pressure regime the film has less dense, more porous morphology at all positions (Fig. 7(c)). This is generally expected for films sputtered at high pressure [32] and it is likely to play a role in the increase of resistivity occurring in this regime. It can also explain the global increase of resistivity over time shown in Fig. 2, since a higher porosity can result in easier adsorption of oxygen and other detrimental chemical species at grain boundaries.

\subsection{Grain size}

The quantity that can be extracted by XRD is the out-of-plane crystallite size, which, especially in the case of columnar growth, is not necessarily related to the in-plane grain size. For this reason, we rely on SEM images in top view to visualize the in-plane grain size and we employ a standard data analysis procedure in XRD line profile analysis to deconvolute micro-strain and instrumental effects from the out-of-plane grain size [19]. Note that this method has been applied to AZO films in a very limited number of previous reports $[33,34,35]$, with most studies not applying any kind of deconvolution. From the SEM images in top view (Fig. 7) it is evident that the in-plane dimensions of the crystal grains decrease from $x_{0}$ to $x_{3}$ at all pressures. On the other hand, the out-of plane grain size measured by XRD (Fig. 8(a)) does not point to a unique trend. In fact, the grain size decreases with increasing radial position in the low- and high pressure regime in a similar fashion as the in-plane grain size, but the opposite is observed in the intermediate pressure regime. Hence, an anomaly exists near $x_{0}$ in the intermediate 
pressure regime. This is qualitatively confirmed by the SEM images in cross-sectional view for such a region (Fig. 7), showing V-shaped grains that do not extend all the way through the film. The origin of this type of morphology will be discussed in the following sections.

Finally, we note that at the $x_{0}$ position in the low pressure regime a striking five-fold increase in grain size occurs with respect to the rest of the film. This corresponds to the position with the most severe amorphization effects at the film top. Further experiments are needed to understand such an abrupt increase. It must be emphasized, however, that this effect would not have been detected without deconvoluting the instrumentaland strain-related XRD line broadening from size-related broadening. In fact, direct grain size estimation with the Scherrer formula before deconvolution yields $24 \mathrm{~nm}$ for this data point, whereas the same formula after deconvolution yields $185 \mathrm{~nm}$. Even though this is an extreme example, skipping the deconvolution step in our data results in an average underestimation of grain size by $43 \%$.

\subsection{Texture}

It is well known that sputter-deposited $\mathrm{ZnO}$ films often exhibit very strong c-axis texture, with the (001) planes of the hexagonal lattice mostly parallel to the plane of the substrate. In this study, we do not carry out a comprehensive texture analysis but we simply evaluate the integrated area of the (002) peak in the XRD pattern, normalized by the film thickness at each point, to give a rough estimate of the degree of preferential c-axis orientation. The results (Fig. 8(b)) demonstrate that completely different radial distributions exist in the different pressure regimes. Notably, the (001) texture is almost completely lost near $x_{0}$ in the intermediate pressure regime. This effect will be discussed in the following sections in conjunction with the trends in grain size.

\section{Discussion}

\subsection{Review of particle energy flux distributions in AZO sputter deposition}

We have concluded that particle bombardment is likely to affect the properties of our AZO film to various extents depending on deposition pressure and radial position. By compiling results from various existing works, we present in the following the expected trends for the total energetic particle flux density and average particle energy with respect to deposition pressure and radial position. The expected trends are then compared to our film characterization results.

4.1.1. Particle flux density The energetic particle flux density dependence on deposition pressure was examined for $\mathrm{DC}$ sputtering of $\mathrm{ZnO}$ [17], where $\mathrm{O}^{-}$ions were found to be the dominating species up to a pressure of about $5 \mathrm{mTorr}$, and $\mathrm{O}$ neutrals dominated above 5 mTorr. The summed flux density from of the two species was only slightly decreasing with increasing pressure up to about 8 mTorr, then a faster 
exponential decay occurred, characterized by a 50\% decrease every 10 mTorr.

The spatial distribution of the $\mathrm{O}^{-}$ion flux density was investigated in several works, which generally found a larger flux density near $x_{e}$, corresponding to the largest plasma density or, equivalently, to the strongest magnetic field at the target $[9,28,41]$. It is difficult to extract quantitative trends, because the flux density distribution depends on the age of the target $[9,42]$, the strength of the magnetic field in the magnetron [10], the type of excitation [9] (RF or DC), and energy-dispersive measurements are typically affected by the limited acceptance angle of the probe [9, 15]. However, it can be assumed that increasing deposition pressure leads to a reduction in the flux density gradient due to a larger contribution from species with an off-normal incidence angle caused by more frequent collisions. This effect can be seen to some extent in angle-resolved flux density measurements [28].

4.1.2. Particle energy The maximum energy of the negative ions at the substrate decreases with increasing deposition pressure, because the particle mean free path decreases with pressure due to collisions in the plasma. However, the decrease is not as strong as might be expected by the classical mean free path of Ar at room temperature, because the collision cross section of energetic species is considerably smaller than that of thermal species [43]. According to universal energy-dependent thermalization curves [44], species with an energy distribution centered at $150 \mathrm{eV}$ (as expected from our measured target self-bias) reach the substrate with still $90 \%, 85 \%$, and $70 \%$ of their initial energy at 2.5 mTorr, 5 mTorr, and 15 mTorr respectively. A rather similar pressure dependence has also been shown experimentally for $\mathrm{O}^{-}$ions in $\mathrm{RF}$ sputtering of $\mathrm{MgO}$ [45].

Regarding the radial dependence, the maximum difference in ballistic path from target to substrate in our setup is from the center of the target to point $x_{0}$ and to point $x_{3}$ on the substrate. Those two distances are $3.0 \mathrm{~cm}$ and $4.2 \mathrm{~cm}$ respectively, i.e. $\mathrm{a} \sim 30 \%$ difference in pressure-distance product. Using the same energy-dependent thermalization curves as above, an upper limit to particle energy gradients can be estimated. From this analysis, we conclude that particle energy at all radial positions should be within $90 \%$ of the energy at the shortest target-substrate path at all investigated pressures. A nearly uniform radial energy distribution is confirmed by existing experimental data [9] and simulations [10, 26].

4.1.3. Comparison with our results The lattice shift trends in our films (Fig. 5(a)) point to the fact that the particle energy flux is enhanced by decreasing the deposition pressure from 15 mTorr to 2.5 mTorr and by decreasing the radial position from $x_{3}$ to $x_{0}$. While the pressure trend is compatible with the above review, the radial trend is not. Specifically, we have indirectly found a maximum in the particle energy flux (and resistivity) at $x_{0}$ through Eq. 1 , in contrast with the typical observation of a maximum at $x_{e}$.

Yet it must be emphasized that previously reported resistivity distributions do 
not necessarily follow the expected energetic particle distribution at the target. While resistivity maxima at $x_{e}$ are most often reported $[9,11,12,7,8,29]$, resistivity minima at $x_{e}$ [13] and resistivity maxima at $x_{0}$ were also observed $[9,11,12,10]$. In some cases $[9,11,12]$, the resistivity maximum at $x_{0}$ was shown to occur only when an old, eroded target was used (roughly, for an erosion track deeper than $1 \mathrm{~mm}$ ). This was related to an increased flux of high-energy oxygen ions in the region around $x_{0}$ due to a modified emission angle distribution of the ions from a target with an eroded profile [9]. However, this mechanism is unlikely to be active in our experiment because our target was nearly uneroded (depth of erosion track: $0.2 \mathrm{~mm}$ ).

Another possible explanation for a resistivity maximum at $x_{0}$ could be the radial inhomogeneity of oxygen reaching the substrate, as clearly shown in a study on DCsputtered $\mathrm{In}_{4} \mathrm{Sn}_{3} \mathrm{O}_{12}$ [18]. There, a maximum at $x_{0}$ occurred when the oxygen content was closer to the optimal amount at $x_{e}$ than at $x_{0}$. However, this is not the case in our experiments either, since the oxygen content in our films is not a monotonic function of radial position (Fig. 6(d)).

Yet a third possible cause for a resistivity maximum at $x_{0}$ could be specific plasma features at the target, influenced by parameters such as strength of the magnetic field, balanced/unbalanced type of cathode, or superposition of a capacitively coupled mode of discharge over the magnetron sputtering regime at low pressure-distance product [46]. For example, it has been shown for DC-sputtered AZO that, upon enhancement of the magnetic field parallel to the target by an external magnetic field, the position of the resistivity maximum shifts from $x_{0}$ to $x_{e}$ [10].

Note also that most previous studies have neglected the detrimental effect of positive ions (of which $\mathrm{Ar}^{+}$are the most abundant) on the basis that their average energy is much lower than that of the $\mathrm{O}^{-}$ions. However, a flux density ratio between $\mathrm{Ar}^{+}$and $\mathrm{O}^{-}$ ions greater than 2 was measured for RF sputtering of AZO with a grounded substrate [27]. In the same study, the average energy of the $\mathrm{Ar}^{+}$ions impinging onto a grounded surface was $29 \mathrm{eV}$, with a significant tail above the $33-35 \mathrm{eV}$ sputtering threshold of $\mathrm{ZnO}[10,26]$. The radial distribution of $\mathrm{Ar}^{+}$ion flux density features a maximum at $x_{0}$, corresponding to a maximum in plasma density at the substrate [9]. Therefore, it is not excluded that $\mathrm{Ar}^{+}$ions may play a role in the the energy flux and resistivity distributions in our experiments.

\subsection{Correlations at low and intermediate deposition pressure}

In this section, we focus on deposition pressures lower than the optimal pressure in Fig. 2(a), i.e., on the left of the resistivity minimum at around 5 mTorr pressure. This corresponds to a process window where the resulting films are under compressive stress. We attempt to identify correlations between the compressive stress and some electrical, structural, and compositional properties. For each pair of correlated properties, we suggest a fitting function for the experimental data, which roughly describes the nature of the correlation. Also, we discuss the possible physical origin of the correlations and 

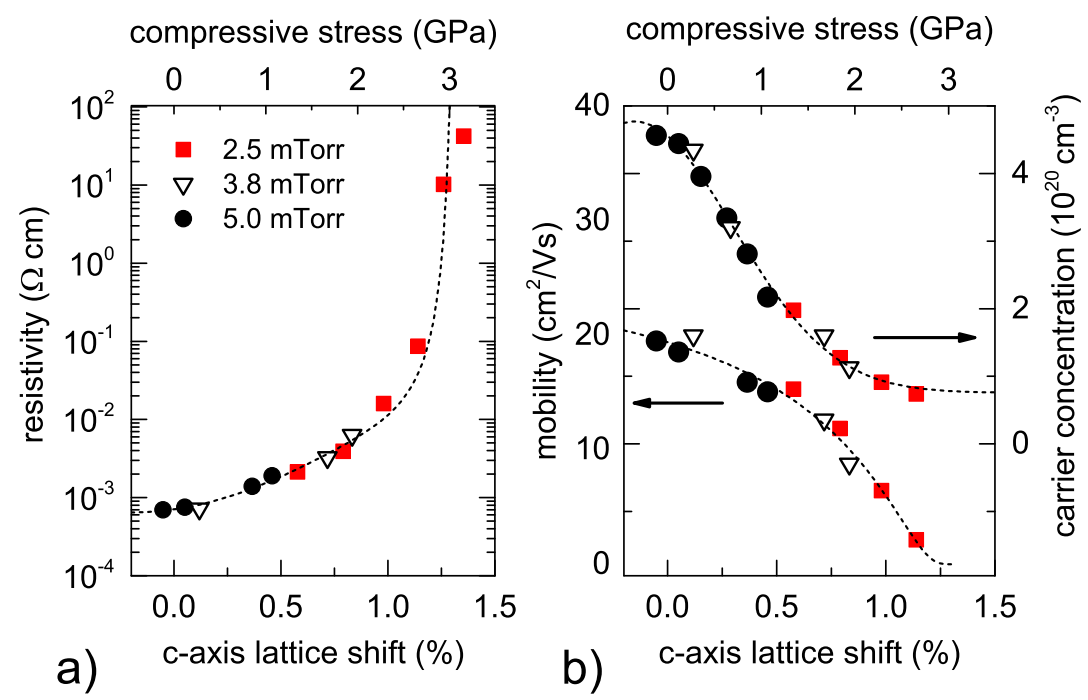

Figure 9. Resistivity, carrier concentration, and mobility of AZO films versus their corresponding lattice shift and stress. The plotted data points are from radial positions between $x_{0}$ and $x_{3}$ at three different deposition pressures in the low- and intermediate pressure regime. The dashed curves are the fitting functions defined by Eqs. 2-4 in the text.

briefly mention the relationship between oxygen content and carrier mobility, which is an additional cause of inhomogeneity in AZO films.

4.2.1. Electrical properties and stress In this study, the electrical properties and the out-of-plane lattice shift (or in-plane compressive stress) of AZO are clearly correlated (Figs. 2 and 5). Since the latter is related to the energy $E$ and flux density $\Gamma$ of the bombarding particles [31] according to Eq. 1, there is strong evidence that particle bombardment has a major influence on the resistivity of RF-sputtered AZO, both in the radial direction and in the low-pressure regime.

To quantify this effect, we propose plotting $y(\bar{p}, \bar{x})$ versus $\varepsilon(\bar{p}, \bar{x})$ curves, where $y$ is any measured quantity at point $(\bar{p}, \bar{x})$ in the pressure-position space. This should be equivalent to plotting quantity $y$ versus a rough measure of the particle energy flux. The results are presented in Fig. 9 for resistivity $\rho$, carrier concentration $N_{c}$, and mobility $\mu$. Strikingly, we find that all three electrical properties can be expressed as a function of the lattice shift alone, regardless of radial position or deposition pressure. By fitting the experimental data points in the three curves in Fig. 9, we obtain the following empirical relations between the electrical properties and the lattice shift:

$$
\begin{aligned}
& N_{c}(\varepsilon)=N_{0}+A_{1} \exp \left(-\frac{\left(\varepsilon-\varepsilon_{1}\right)^{2}}{2 B_{1}^{2}}\right) \\
& \mu(\varepsilon)=A_{2} \exp \left(\frac{B_{2}}{\varepsilon-\varepsilon_{2}}\right)
\end{aligned}
$$




$$
\rho(\varepsilon)=\frac{1}{q A_{2} \exp \left(\frac{B_{2}}{\varepsilon-\varepsilon_{2}}\right)\left(N_{0}+A_{1} \exp \left(-\frac{\left(\varepsilon-\varepsilon_{1}\right)^{2}}{2 B_{1}^{2}}\right)\right)}
$$

where Eq. 4 was simply derived by applying the relation $\rho=\left(q N_{c} \mu\right)^{-1}$, $q$ being the elementary charge. The fitted values of the free parameters are: $N_{0}=7.57 \times 10^{19} \mathrm{~cm}^{-3}$, $A_{1}=3.01 \times 10^{20} \mathrm{~cm}^{-3}, \varepsilon_{1}=-0.151 \%, B_{1}=0.465 \%, A_{2}=28.9 \mathrm{~cm}^{2} / \mathrm{Vs}, \varepsilon_{2}=1.34 \%$, $B_{2}=0.537 \%$.

These results suggest that the lattice shift (and ultimately the energy flux of the bombarding particles) is correlated to the electrical properties in a more general way than the single deposition parameters or the radial position are. Namely, a certain value of the lattice shift may be obtained by different combinations of radial position, deposition pressure and possibly other parameters, but the resistivity is ultimately determined by the lattice shift resulting from those deposition parameters. It is evident from Fig. 9(a) that lattice shift must be minimized in order to minimize the film resistivity. This can be achieved by minimizing the energy flux of bombarding particles as demonstrated, for example, by placing the substrate perpendicularly to the target $[47,48]$ or by a variety of other methods [16]. Note also that, in the case of a large compressive stress, the carrier concentration stabilizes to its asymptotic value given by $N_{0}$, while the mobility drops dramatically.

4.2.2. Structural properties and stress The out-of-plane grain size and the degree of preferential c-axis orientation are also correlated with compressive stress and thus with the particle energy flux (Fig. 10(a)). In this case, however, the correlation is only qualitative and the fitting functions are merely a guide to the eye. Both the grain size and (002) peak area have a minimum at a compressive stress of about 1-2 GPa. This is likely to correspond to the energy flux and temperature window typical of zone $\mathrm{T}$ in the Thornton structure zone diagram [32], where a mixed film texture and small out-of plane grain size are expected due to competition between differently oriented V-shaped grains during film growth.

Here an interesting observation can be made. Most of the existing studies on sputtered AZO report an increase in grain size [7, 49, 9] and an increase in (001) texture coefficient [50] with decreasing resistivity, but inconsistent trends have been pointed out, especially for the case of RF sputtering [9, 51, 33]. From inspection of Fig. 10(a), we propose that the often-reported trend of increasing grain size with decreasing resistivity applies only under mild particle bombardment, i.e. for $\sigma<1 \mathrm{GPa}$, where resistivity is lowest. This possibly corresponds to zone 1 in the Thornton zone diagram. Conversely, the grain size increases for increasing energy flux and increasing resistivity when $\sigma>2 \mathrm{GPa}$, as the film structure possibly moves to zone 2 of the diagram. This may provide an explanation for the reported inconsistencies in the literature. In fact, the lattice shift in one study that reported the anomalous trend of increasing grain size with increasing resistivity was in the $1.0-1.5 \%$ range [9], where that trend is actually predicted by our qualitative model. 

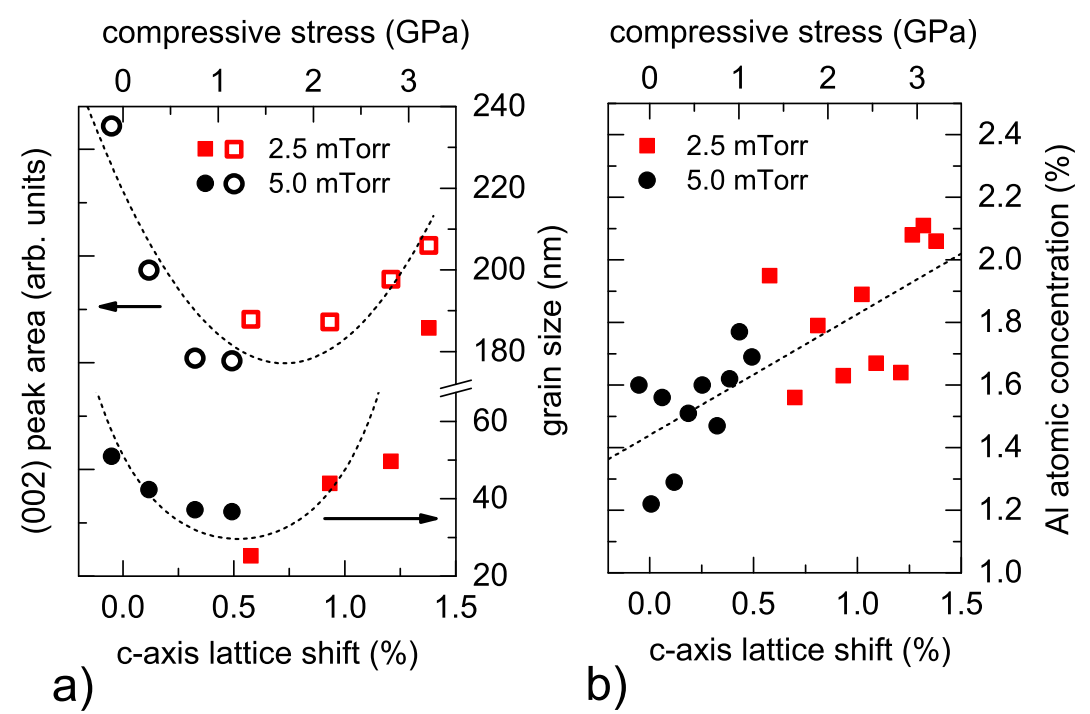

Figure 10. (a) Area of (002) peak and out-of-plane grain size of AZO films versus their corresponding lattice shift and stress. The plotted data points are from radial positions between $x_{0}$ and $x_{3}$ at two different deposition pressures in the low- and intermediate pressure regime. The dashed curves are fitting functions to be interpreted only qualitatively. (b) Atomic $\mathrm{Al}$ concentration at different radial positions in the lowand intermediate pressure regime versus their corresponding lattice shift and stress. The dashed curve is the fitting function defined by Eq. 5 .

4.2.3. Aluminum content and stress While it is difficult to establish any trends in the $\mathrm{Al}$ content against radial position alone (Fig. $6(\mathrm{~b})$ ), the $\mathrm{Al}$ content $c_{\mathrm{Al}}$ seems to increase roughly linearly with lattice shift (Fig. 10(b)) and can be fitted as

$$
c_{\mathrm{Al}}(\varepsilon)=A_{3}+B_{3} \varepsilon
$$

where $A_{3}=1.44 \%$ and $B_{3}=0.387$. Al enrichment has been observed by several authors when the substrate temperature was increased above an optimal value $[52,37,53]$. Even though the substrate temperature was kept constant in this study, the actual temperature of the growing film is given by the substrate temperature plus a temperature shift proportional to the potential energy flux of the particles reaching the substrate. The latter depends on the number flux density of the species, their cohesive and ionization energy and work function, but not on their kinetic energy [40]. However, the potential energy flux is still expected to increase for decreasing pressure (due to less thermalization) [54] and for decreasing radial position (due to higher deposition rate, reflecting the higher number flux density of all species arriving at the substrate) similarly to the kinetic energy flux and the lattice shift [46]. Hence we may interpret the increase in $\mathrm{Al}$ content with lattice shift as due to a film temperature increase related to the increased potential energy flux density at the substrate.

4.2.4. Mobility and oxygen content Besides the global resistivity maximum at $x_{0}$, weaker local maxima of the Hall mobility are also observed near $x_{e}$ in the intermediate 
pressure regime (Fig. 3(b)). We suggest that a correlation exists between the local Hall mobility maxima and the maxima of the oxygen content near $x_{e}$ found in the intermediate pressure regime (Fig. 6(d)). If this hypothesis is true, the oxygen content in the other regions of the film must be slightly below the optimal value. Note, however, that the measured change in oxygen content near $x_{e}$ is less than $+0.5 \%$ (absolute), pointing to the high sensitivity of electron mobility on oxygen content. A local maximum in oxygen content is also present, at slightly different radial positions, in the low- and high-pressure regimes, which however do not exhibit an evident mobility spike. We presume that the mobility change is harder to detect in those regimes due to the steeper gradient or lower value of the mobility.

\subsection{Correlations at high deposition pressure}

In this section, we focus on deposition pressures higher than the optimal pressure in Fig. 2(a), i.e. on the right of the resistivity minimum at around 5 mTorr pressure. This corresponds to a process window where the resulting films are under tensile stress. No quantitative correlations were found in this process window, so a purely qualitative discussion is provided.

4.3.1. Electrical properties and porosity SEM images confirm that a less dense, more porous film is formed in the high pressure regime (Fig. $7(\mathrm{c})$ ), accompanied by tensile in-plane stress (Fig. 5(a)). This can be attributed to thermalization of most sputtered species. In fact, the mean free path for neutrals $(\mathrm{Zn}, \mathrm{O}, \mathrm{Al})$ is around $2.0 \mathrm{~cm}$ at 2.5 mTorr, comparable to our target-substrate distance, but it is only around $3.4 \mathrm{~mm}$ at 15 mTorr [43]. Porosity can explain the inferior electrical properties in the as-deposited films, because the electrical current has to flow around a larger number of voids. Since more grain boundaries are exposed to air than in an ideally compact film, a porous morphology can also explain the time-dependent degradation under ambient conditions (Fig. 2(b)) due to adsorbed species at grain boundaries. However, it is unlikely that the resistivity gradient in the radial direction is also due to an increase in porosity from $x_{3}$ to $x_{0}$. First of all, film density is more likely to increase near $x_{0}$, rather than the opposite, due to a larger expected energy flux of the bombarding particles, which is confirmed by a lower tensile stress in that region. Secondly, no significant difference in the refractive index and extinction coefficient was measured from from $x_{3}$ to $x_{0}$ (not shown), suggesting that film density is approximately constant in the radial direction. Finally, the resistivity degradation factor under ambient exposure is approximately constant in the radial direction (Fig. 2(a)), which also suggests that the density gradient is negligible. 


\section{Conclusion}

We have investigated the spatial distribution of different electrical, structural, morphological, and compositional properties of RF-sputtered AZO at different deposition pressures. Based on a well-known relationship between the kinetic energy flux of the bombarding particles and the compressive stress in the film, we have correlated several properties of the films to the energy flux. Generalized quantitative relationships have been established between the electrical properties (carrier concentration, mobility, and resistivity) and the compressive stress in the film. In general, the stress must be as low as possible in order to minimize resistivity. At a large stress (above $2 \mathrm{GPa}$ ) the carrier concentration stabilizes to an approximately constant value while the mobility drops dramatically.

On the other hand, preferential c-axis orientation and grain size both have a very different relationship to the compressive stress and the bombarding energy flux. They

have a minimum at a compressive stress in the 1-2 GPa range, where the most random orientation and the smallest grain size are found, corresponding to zone $\mathrm{T}$ of the Thornton structure zone diagram. Moving away from this stress range, the grains increase in size and the preferential (001) orientation is restored. This can explain why inconsistent correlations between resistivity and grain size have often been found in previous work.

The $\mathrm{Al}$ content in the film also has an (approximately linear) relationship to the compressive stress. We attribute the $\mathrm{Al}$ enrichment to an increased potential energy flux from the species arriving at the substrate, resulting in a temperature increase of the growing film.

From the analysis of deposition rate, film morphology and stress, it is concluded that the main reason for spatial inhomogeneity and pressure dependence of the electrical properties is particle bombardment. However, we have also observed localized gradients in the electron mobility (but not in the carrier concentration) in an intermediate pressure regime, which we attributed to inhomogeneous oxygen distribution. While this was found to be a minor effect under our deposition conditions, oxygen inhomogeneity may become a dominating factor if films with a uniform low-stress profile can be produced at a reasonably low pressure.

Finally, under process conditions where particle bombardment effects are negligible and in-plane tensile stress develops, a porous microstructure limits the achievable resistivity and causes it to increase over time as well.

\section{Acknowledgments}

This work has been supported by a grant from the Danish Council for Strategic Research. CINF is funded by the Danish National Research Foundation (DNRF54). A.C. is grateful to Edoardo Bosco for four-point probe measurements and to Karen and Wolfgang Pantleon for assistance with XRD line profile analysis. 


\section{References}

[1] Ellmer K 2012 Nat. Photonics 6 809-817

[2] Kumar A and Zhou C 2010 ACS Nano 4 11-4

[3] Green M A, Emery K, Hishikawa Y, Warta W and Dunlop E D 2015 Prog. Photovolt: Res. Appl. 24 3-11

[4] Herrmann D, Kratzert P, Weeke S, Zimmer M, Djordjevic-Reiss J, Hunger R, Lindberg P, Wallin E, Lundberg O and Stolt L 2014 CIGS module manufacturing with high deposition rates and efficiencies 2014 IEEE 40th Photovoltaic Specialist Conference (PVSC) (IEEE) pp 2775-2777

[5] Jackson P, Hariskos D, Wuerz R, Kiowski O, Bauer A, Friedlmeier T M and Powalla M 2015 Phys. Status Solidi RRL 9 28-31

[6] Ellmer K 2000 J. Phys. D: Appl. Phys. 33 R17-R32

[7] Tominaga K, Yuasa T, Kume M and Tada O 1985 Jpn. J. Appl. Phys. $24944-949$

[8] Minami T, Miyata T, Yamamoto T and Toda H 2000 J. Vac. Sci. Technol., A 181584

[9] Bikowski A, Welzel T and Ellmer K 2013 J. Appl. Phys. 114223716

[10] Zhang X B, Pei Z L, Gong J and Sun C 2007 J. Appl. Phys. 101014910

[11] Szyszka B 2008 Magnetron Sputtering of ZnO Films Transparent Conductive Zinc Oxide: Basics and Applications in Thin Film Solar Cells ed Ellmer K, Klein A and Rech B (Berlin: Springer) p 221

[12] Dewald W, Sittinger V, Werner W, Jacobs C and Szyszka B 2009 Thin Solid Films 518 $1085-1090$

[13] Cebulla R, Wendt R and Ellmer K 1998 J. Appl. Phys. 831087

[14] Tominaga K, Sueyoshi Y, Munfei C and Shintani Y 1993 Jpn. J. Appl. Phys. 32 4131-4135

[15] Welzel T, Kleinhempel R, Dunger T and Richter F 2009 Plasma Processes Polym. 6 S331-S336

[16] Ellmer K and Welzel T 2012 J. Mater. Res. 27 765-779

[17] Tominaga K, Iwamura S, Shintani Y and Tada O 1982 Jpn. J. Appl. Phys. 21 688-695

[18] Minami T, Takeda Y, Takata S and Kakumu T 1997 Thin Solid Films 308-309 13-18

[19] de Keijser T H, Langford J I, Mittemeijer E J and Vogels A B P 1982 J. Appl. Crystallogr. 15 308-314

[20] Petersen D H, Hansen O, Lin R and Nielsen P F 2008 J. Appl. Phys. 104013710

[21] Henrichsen H H, Hansen O, Kjaer D, Nielsen P F, Wang F and Petersen D H 2014 Precision of single-engage micro Hall effect measurements 2014 International Workshop on Junction Technology (IWJT) (IEEE) pp 1-4

[22] Kluth O, Schöpe G, Rech B, Menner R, Oertel M, Orgassa K and Werner Schock H 2006 Thin Solid Films 502 311-316

[23] Chung Y M, Moon C S, Jung M J and Han J G 2005 Surf. Coat. Technol. 200 936-939

[24] Ngaruiya J M, Kappertz O, Mohamed S H and Wuttig M 2004 Appl. Phys. Lett. 85748

[25] Keller J H and Simmons R G 1979 IBM J. Res. Dev. 23 24-32

[26] Carcia P F, McLean R S, Reilly M H, Li Z G, Pillione L J and Messier R F 2003 J. Vac. Sci. Technol., A 21745

[27] Welzel T and Ellmer K 2013 J. Phys. D: Appl. Phys. 46315202

[28] Tominaga K, Ueshiba N, Shintani Y and Tada O 1981 Jpn. J. Appl. Phys. 20 519-526

[29] Cai Y, Liu W, He Q, Zhang Y, Yu T and Sun Y 2010 Appl. Surf. Sci. 256 1694-1697

[30] DHeurle F M 1970 Metall. Mater. Trans. B 1 725-732

[31] Windischmann H 1987 J. Appl. Phys. 621800

[32] Thornton J A 1977 Annu. Rev. Mater. Sci. 7 239-260

[33] Charpentier C, Prodhomme P, Maurin I, Chaigneau M and Roca i Cabarrocas P 2012 EPJ Photovoltaics 225002

[34] Charpentier C, Prod'homme P and Roca i Cabarrocas P 2013 Thin Solid Films 531 424-429

[35] Bikowski A and Ellmer K 2012 J. Mater. Res. 27 2249-2256

[36] Ellmer K, Kudella F, Mientus R, Schieck R and Fiechter S 1994 Thin Solid Films 247 15-23

[37] Cornelius S and Vinnichenko M 2015 Thin Solid Films, http://dx.doi.org/10.1016/j.tsf.2015.11.059 
[38] Agashe C, Kluth O, Schöpe G, Siekmann H, Hüpkes J and Rech B 2003 Thin Solid Films 442 $167-172$

[39] Mattox D M 1989 J. Vac. Sci. Technol., A 71105

[40] Anders A 2010 Thin Solid Films 518 4087-4090

[41] Toyoda H, Goto K, Ishijima T, Morita T, Ohshima N and Kinoshita K 2009 Appl. Phys Express 2126001

[42] Welzel T and Ellmer K 2011 Surf. Coat. Technol. 205 S294-S298

[43] Harper J M E, Cuomo J J, Gambino R J, Kaufman H R and Robinson R 1978 J. Vac. Sci. Technol. 151597

[44] Somekh R E 1984 J. Vac. Sci. Technol., A 21285

[45] Ishijima T, Goto K, Ohshima N, Kinoshita K and Toyoda H 2009 Jpn. J. Appl. Phys. 48116004

[46] Musil J 1998 Vacuum 50 363-372

[47] Minami T, Sato H, Nanto H and Takata S 1985 Jpn. J. Appl. Phys. 24 L781-L784

[48] Jin Z C, Hamberg I and Granqvist C G 1988 J. Appl. Phys. 645117

[49] Minami T, Nanto H, Sato H and Takata S 1988 Thin Solid Films 164 275-279

[50] Prabhakar T, Dai L, Zhang L, Yang R, Li L, Guo T and Yan Y 2014 J. Appl. Phys. 115083702

[51] Jeong S and Boo J 2004 Thin Solid Films 447-448 105-110

[52] Vinnichenko M, Gago R, Cornelius S, Shevchenko N, Rogozin A, Kolitsch A, Munnik F and Moller W 2010 Appl. Phys. Lett. 96141907

[53] Bikowski A, Rengachari M, Nie M, Wanderka N, Stender P, Schmitz G and Ellmer K 2015 APL Mater. 3060701

[54] Sahu B B, Han J G, Hori M and Takeda K 2015 J. Appl. Phys. 117023301 